\title{
IFN- $\tau$ Mediated Control of Bovine Major Histocompatibility Complex Class I Expression and Function via the Regulation of bta-miR-148b/152 in Bovine Endometrial Epithelial Cells
}

\author{
Haichong Wu, Kangfeng Jiang, Shuai Guo, Jing Yang, Gan Zhao, Changwei Qiu \\ and Ganzhen Deng*
}

Department of Clinical Veterinary Medicine, College of Veterinary Medicine, Huazhong Agricultural University, Wuhan, China

\section{OPEN ACCESS}

Edited by:

Florent Ginhoux

Singapore Immunology Network

(A*STAR), Singapore

Reviewed by:

Alice Sijts,

Utrecht University, Netherlands

Shouxiong Huang,

University of Cincinnati, United States

*Correspondence:

Ganzhen Deng

ganzhendeng@sohu.com

Specialty section:

This article was submitted to Antigen

Presenting Cell Biology,

a section of the journal

Frontiers in Immunology

Received: 15 June 2017

Accepted: 18 January 2018

Published: 02 February 2018

Citation:

Wu H, Jiang K, Guo S, Yang J, Zhao G, Qiu C and Deng G (2018)

IFN- $\tau$ Mediated Control of Bovine Major Histocompatibility Complex

Class I Expression and Function via the Regulation of bta-miR-148b/152 in Bovine Endometrial Epithelial Cells.

Front. Immunol. 9:167.

doi: 10.3389/fimmu.2018.00167
IFN- $\tau$, a type I interferon produced by the trophoblasts of ruminants, has various important immune functions, including effects on the expression of major histocompatibility complex (MHC) class I (MHC-I). A previous study has reported that IFN- $\tau$ promotes the expression of MHC-I molecules on endometrial cells. However, the immunological mechanisms by which IFN- $\tau$ regulates MHC-I molecules remain unknown. Here, we investigated which microRNA (miRNAs) may be involved in the regulation of MHC-I molecule expression and function in bovine endometrial epithelial cells (bEECs). By using TargetScan 6.2 and http://www.microRNA.org, two miRNAs were suggested to target the 3'UTR of the bovine MHC-I heavy chain: bta-miR-148b and bta-miR-152. Dual luciferase reporter and miRNA mimic/inhibitor assays suggested that bta-miR148b/152 were negatively correlated with bovine MHC-I heavy chain genes. The function of the MHC-I heavy chain was then investigated using qRT-PCR, ELISA, western blotting, immunofluorescence, and RNA interference assays in primary bEECs and an endometrial epithelial cell line (BEND). The results demonstrated that bta-miR-148b/152 could promote TLR4-triggered inflammatory responses by targeting the bovine $\mathrm{MHC}-\mathrm{I}$ heavy chain, and the MHC-I molecule negatively regulated TLR4-induced inflammatory reactions may through the Fps-SHP-2 pathway. Our discovery offers novel insight into negative regulation of the TLR4 pathway and elucidates the mechanism by which bovine $\mathrm{MHC}-\mathrm{I}$ molecules control congenital inflammatory reactions.

Keywords: IFN- $\tau$, major histocompatibility complex class I, TLR4, bta-miR-148b/152, bovine endometrial epithelial cells

\section{INTRODUCTION}

IFN, a type I interferon, is secreted by ruminant animal trophoblasts (1). It is responsible for maternal recognition of pregnancy in ruminants, which links the fields of developmental biology and reproductive immunology (2). Many experiments have shown that IFN- $\tau$ also has multiple biological functions, such as anti-proliferative, anti-inflammatory, and antiviral effects (3-5). The bovine major histocompatibility complex (MHC), also called the bovine leukocyte antigen (BoLA), is involved in the regulation of the immune response through antigen recognition by T lymphocytes (6). The MHC class I (MHC-I) region in mammals contains both classical and non-classical MHC-I genes (7). Classical MHC-I molecules, such as the MHC-I heavy chain, play important roles in the immune 
response $(8,9)$. Several studies have shown that IFN- $\tau$ promotes MHC-I expression on endometrial cells (10). However, how the immunological mechanisms of IFN- $\tau$ regulate MHC-I molecules remains unknown. Thus, the high-throughput sequencing technology was employed to investigate the specific microRNA (miRNA) library of IFN- $\tau$ on bovine endometrial epithelial cells (bEECs) cells (11).

MicroRNAs, which are endogenous RNAs of approximately 22 nucleotides, play major roles in the regulation of plants and animals via targeting mRNAs for disruption or translational repression (12). Previous research has indicated that miRNA plays an essential role in various physiological and pathological processes, including development, proliferation, and differentiation (13). There is some evidence to suggest that miR-148/152 specifically mediates HLA-G expression and other immune responses $(14,15)$. However, it is unknown whether any other

TABLE 1 | Primers used for qPCR.

\begin{tabular}{|c|c|c|c|}
\hline Name & $\begin{array}{l}\text { Sequence }\left(5^{\prime} \rightarrow 3^{\prime}\right) \text { : } \\
\text { forward and reverse }\end{array}$ & Accession no. & $\begin{array}{l}\text { Product size } \\
\text { (bp) }\end{array}$ \\
\hline $\begin{array}{l}\text { MHC class I } \\
\text { heavy chain }\end{array}$ & $\begin{array}{l}\text { TATGTGGACGACACGCAGT } \\
\text { TCGCTCTGGTTGTAGTAGCC }\end{array}$ & $\begin{array}{l}\mathrm{NM}_{-} \\
001038518.2\end{array}$ & 188 \\
\hline$\beta$-Actin & $\begin{array}{l}\text { СTCTTCCAGCCTTCCTTCCT } \\
\text { GGGCAGTGATCTCTITCTGC }\end{array}$ & BC102948 & 124 \\
\hline
\end{tabular}

miRNAs are involved in the regulation of bovine MHC-I molecules. Therefore, the primary purpose of this research was to identify candidate miRNAs that may regulate bovine MHC-I molecule expression and function.

Toll-like receptor (TLR) is a type I transmembrane protein with ectodomains containing leucine-rich repeats that recognizes different pathogen-associated molecular patterns and plays vital roles in host defenses against invading microbial pathogens $(16,17)$. TLR4 was the first identified mammalian TLR (18). It regulates immunologic responses against host molecules responsible for tissue injury and chronic inflammation (19). TLR4 is an important receptor for bacterial lipopolysaccharide (LPS) (20). After the binding of LPS, which triggers the activation of the myeloid differentiation factor 88 (MyD88)-dependent pathway, the nuclear transcription factor (NF)- $\kappa \mathrm{B}$ stimulates inflammatory cytokine secretion $(21,22)$. A previous study showed that MHC-I molecules interact with TLR ligands and then suppress innate inflammatory responses (23).

Considering that miRNA is a highly conserved regulatory molecule in many different species, it is possible that the bovine MHC-I heavy chain is regulated by miR-148/152. By using TargetScan 6.2 and http://www.microRNA.org, two miRNAs were suggested to target the 3'UTR of the bovine MHC-I heavy chain: bta-miR-148b and bta-miR-152. In the current research, we investigated which one(s) of these miRNAs may be involved in the

\section{A}
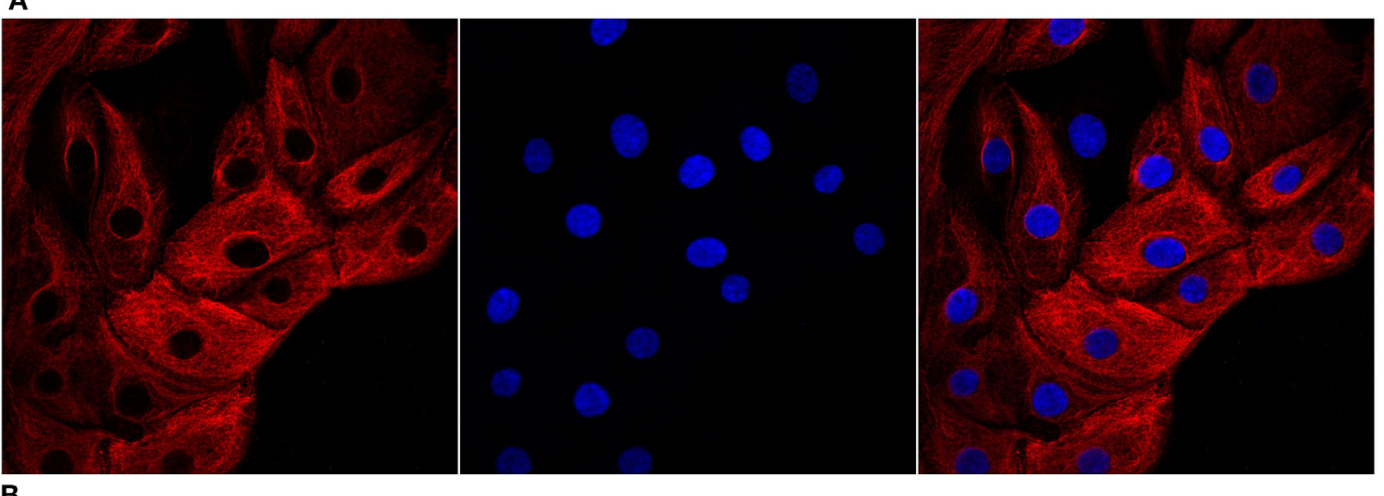

B
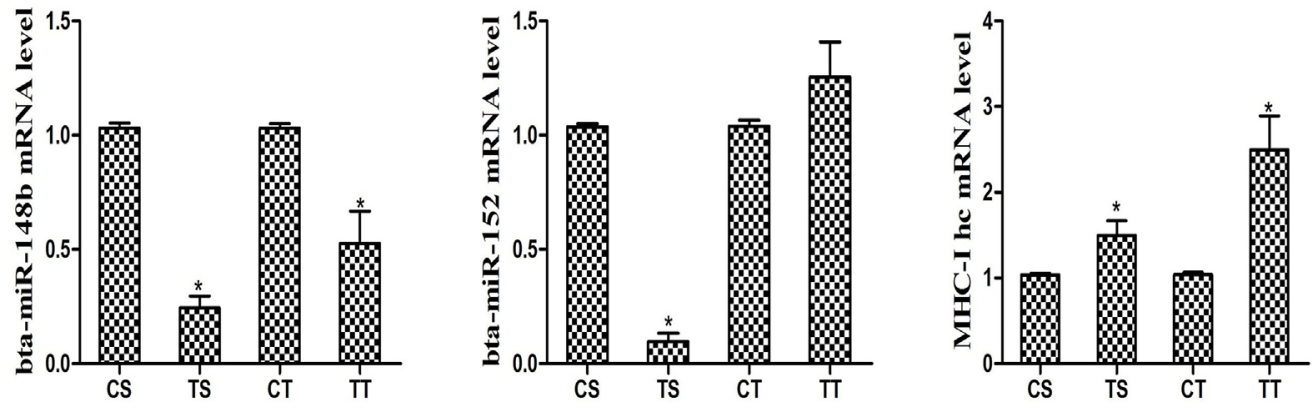

FIGURE 1 | Cell identification and expression of the MHC class I (MHC-I) heavy chain and bta-miR-148b/152 in IFN- $\tau$ stimulated cells. (A) Bovine endometrial epithelial cells were pretreated with fluorochrome to observe endometrial epithelial cell integrity. The cell nucleus was marked with blue fluorescence. Cytokeratin 18 was labeled with a red fluorochrome (magnification 400x). (B) Relative bta-miR-148b/152 and MHC-I heavy chain mRNA expression levels in IFN- $\tau$-stimulated cells were determined by qRT-PCR analysis. U6 was used as an internal control. The TS and TT groups were challenged with IFN- $\tau$ for 6 or 12 h. The CS and CT groups were untreated cells and were used as control groups at the corresponding time points of 6 and $12 \mathrm{~h}$. Data are presented as the mean \pm SEM of three independent experiments. ${ }^{\star} P<0.05$ versus the control group. 
regulation of MHC-I molecule expression and function in bEECs. We demonstrated that down-regulated bta-miR-148b/152 could suppress LPS-triggered inflammatory responses by targeting the bovine MHC-I heavy chain, and the MHC-I molecule negatively regulated TLR4-induced inflammatory reactions may through the Fps-SHP-2 pathway. Therefore, bta-miR-148b/152 may act in the fine tuning of TLR4-triggered innate inflammatory responses.

\section{MATERIALS AND METHODS}

\section{Reagents}

Recombinant bovine interferon-tau (IFN- $\tau$, HPLC >97\%) was purchased from Creative Bioarray (NY, USA). The commercial bovine endometrial cell line (BEND) was obtained from American Type Culture Collection (USA). The Anti-HLA Class I antibody (W6/32) was obtained from abcam (ab23755, UK). BEND cells were cultured and propagated as described in the instructions provided by ATCC.

\section{Cell Culture, Identification, and Treatment}

Primary bEECs were detached and cultivated as described previously (24). Briefly, the uterus of Holstein cow was collected from a local slaughterhouse and immediately taken back to the laboratory in phosphate-buffered solution (PBS). Then, the uterus was cut up, digested with collagenase-I for $2 \mathrm{~h}$, neutralized, and placed in a Petri dish. Cells were cultivated in DMEM/F12 containing 10\% FBS and incubated with 5\% $\mathrm{CO}_{2}$ at $37^{\circ} \mathrm{C}$

The cells were passaged in 12 -well plates with cover slips, and we analyzed the expression of the epithelial-specific marker cytokeratin 18. Cells were fixed in formalin for $10 \mathrm{~min}$ and then washed three times with PBS. The cells were blocked with $10 \%$ normal goat serum (Invitrogen, USA) at room temperature for $30 \mathrm{~min}$ and then incubated with a primary cytokeratin 18 antibody (diluted 1:300 in PBS) overnight at $4^{\circ} \mathrm{C}$. The secondary fluorescently labeled antibody, Dylight 594 antibodies (Bioss, China), was incubated with the cells for $45 \mathrm{~min}$ at room temperature. DAPI was used to stain the cell nuclei. Fluorescence images were obtained using confocal microscopy (Leica, Germany).

The bEECs were plated at $1 \times 10^{6} / \mathrm{mL}$ in six-well plates and then challenged with IFN- $\tau$ ( $200 \mathrm{ng} / \mathrm{mL}$ ) for 6 (TS group) or $12 \mathrm{~h}$ (TT group); untreated cells were used as control groups at the corresponding time points of 6 and $12 \mathrm{~h}$ (CS and CT groups, respectively).

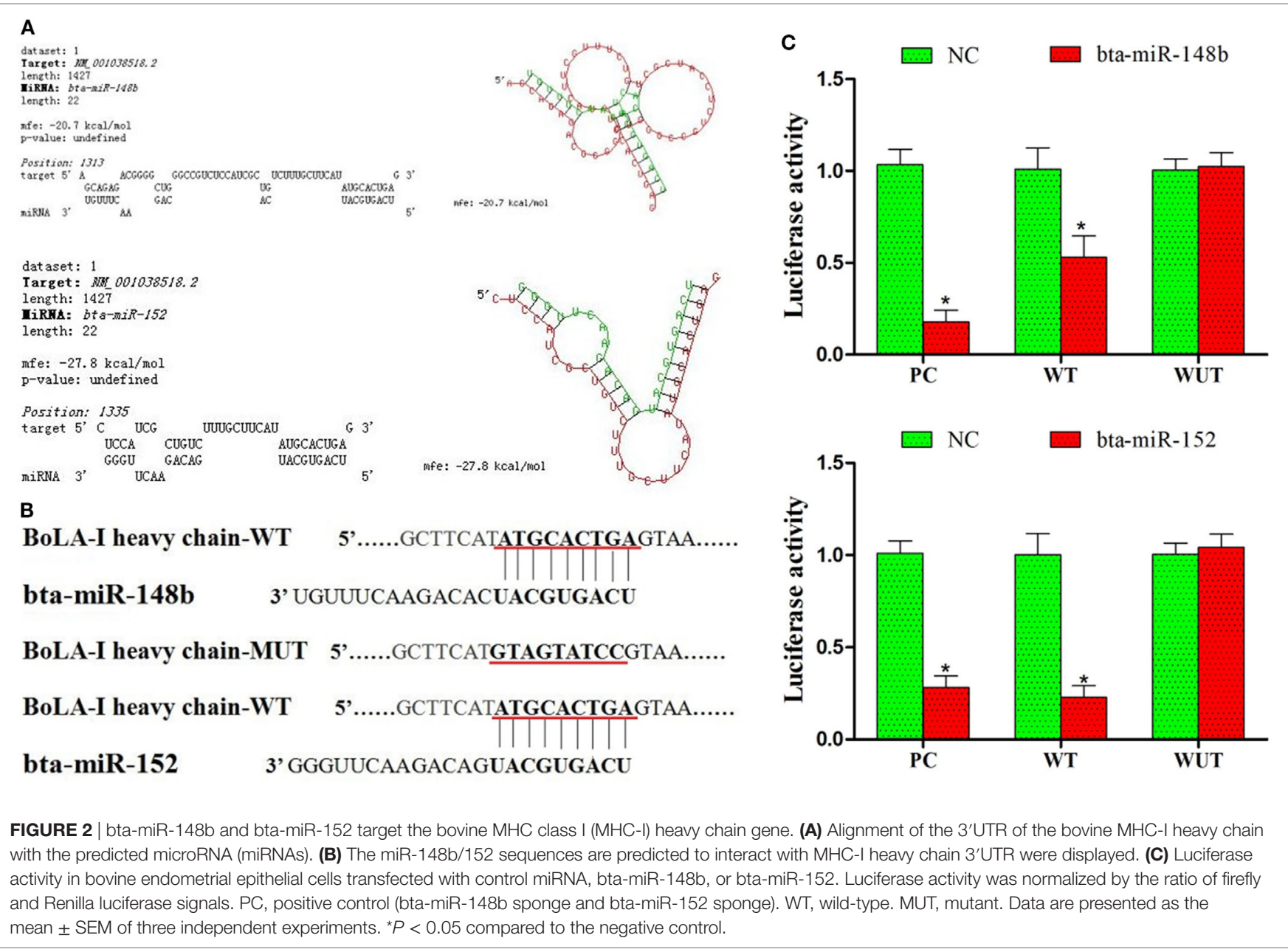




\section{qRT-PCR Analysis}

Total RNA was isolated by TRIzol agent following the producer's directions (Invitrogen, USA). PCR was carried out with a SYBR ${ }^{\circledR}$ Select Master Mix kit and PCR amplification system. The primers are listed in Table 1. The expression levels of related genes were standardized to $\beta$-actin expression using the $2^{-\Delta \Delta \mathrm{Ct}}$ comparative method. For miRNA qRT-PCR, a commercial Hairpin-it ${ }^{\mathrm{TM}}$ miRNAs qPCR Quantification kit (GenePharma, China) was used. Specific primers for bta-miR-148b, bta-miR-152, and U6 were designed by GenePharma. qRT-PCR was carried out using a SYBR ${ }^{\circledR}$ Select Master Mix kit and PCR amplification system. The expression of miRNAs was standardized to U6 expression using the $2^{-\Delta \Delta C t}$ comparative method.

\section{Transfection with miRNA Mimics and Inhibitors}

The bEECs were transfected with $50 \mathrm{nM}$ bta-miR-148b and btamiR-152 mimics, inhibitors, or corresponding negative control constructs (GenePharma, China) using Lipofectamine 2000 following the producer's directions. Transfected cells were harvested at $24 \mathrm{~h}$.

\section{Cytokine Assay}

The concentrations of TNF- $\alpha$ and IL- $1 \beta$ in the supernatant of cells were detected with ELISA kits according to the producer's directions. The optical density value was acquired at $450 \mathrm{~nm}$ with a microplate reader.

\section{Plasmid Construction}

The predicted target sites of the $3^{\prime}$-UTR of the bovine MHC-I heavy chain gene or sequences containing mutated versions of the predicted target sites were cloned into the SacI and XhoI sites of the pGLO promoter vector (Promega, USA). The bta-miR-148b/152 target site $5^{\prime}$-ATGCACTGA-3' in the bovine MHC-I heavy chain $3^{\prime}$-UTR was mutated to $5^{\prime}$-GTAGTATCC-3' by site-directed mutagenesis. To prepare bta-miR-148b/152-GLO vectors, PCR amplification was performed on bta-miR-148b and bta-miR-152 sponges. The PCR products were digested with SacI and XhoI and cloned into the pmirGLO vector. The primers were as follows: btamiR-148b sponge sense $5^{\prime}$-CACAAAGTTCTGTGATGCACTGA ATCGACAAAGTTCTGTGATGCAC TGAC-3' and antisense 5' - TCGAGTCAGTGCATCACAGAACTTTGTCGATTCAG TGCATCACAGAACTTTGTGAGCT-3'; bta-miR-152 sponge sense 5'-CCCCGTTC TGTCATGCACTGAATCGCCCAAGT TCTGTCATGCACTGAC-3' ${ }^{\prime}$, and antisense $5^{\prime}$-TCGAGTCAGTG CATGACAGAACTTGGGCGATTCAGTGCATGACAGA ACTTGGGGAGCT-3'.

\section{Luciferase Activity Analysis}

HEK-293 cells were cultivated in DMEM containing 10\% FBS in 24-well dishes with $5 \% \mathrm{CO}_{2}$ at $37^{\circ} \mathrm{C}$. Cells were transfected at $80 \%$ confluency with $2 \mu \mathrm{L}$ of lipofectamine 2000 (Invitrogen, USA) in $100 \mu \mathrm{L}$ of Opti-MEM I without serum, following the lipofectamine producer's directions. Each plate was transfected with $80 \mathrm{ng}$ of pmirGLO-MHC-I heavy chain/WT vector or pmirGLO-MHC-I heavy chain/MUT vector containing firefly luciferase and the pRLTK vector (Promega, USA) containing bta-miR-148b-GLO,
bta-miR-152-GLO, or control-GLO (pmirGLO, Promega). Cells were then cultured for $24 \mathrm{~h}$ without changing the medium and were lysed for 15 min with $30 \mu \mathrm{L}$ of Passive Lysis Buffer (Promega, USA) on a rocking platform. Firefly luminescence was normalized to renilla luminescence to calculate the relative luciferase activity using the Dual Luciferase Reporter Assay following the producer's directions.

\section{Western Blot Analysis}

Cells were lysed with lysis buffer containing phosphatase inhibitor and centrifuged at $10,000 \mathrm{~g}$ for $10 \mathrm{~min}$. The protein concentration

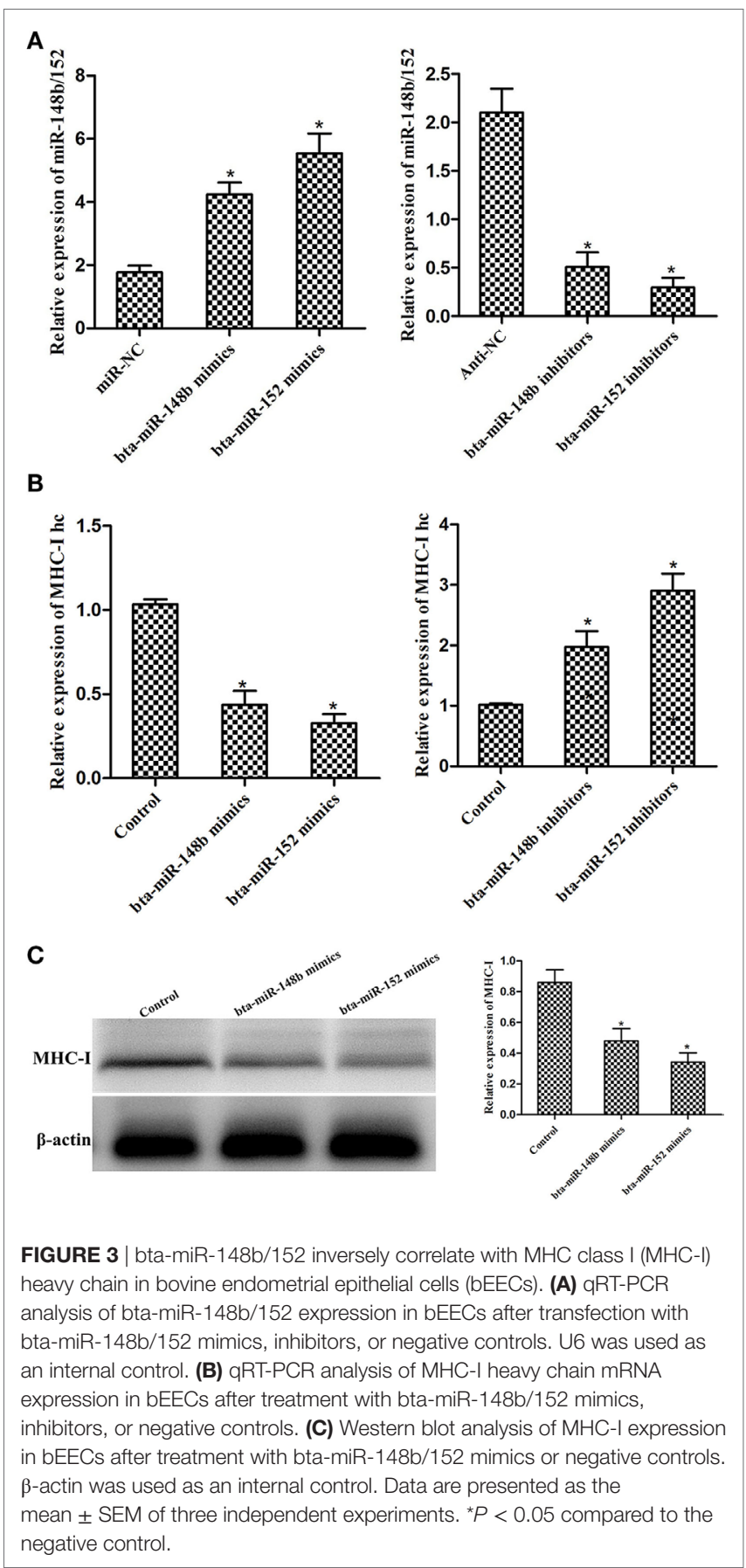


was determined with a BCA kit. Then, the same amount of protein from each sample was separated by SDS-PAGE and transferred onto a PVDF membrane. The membrane was incubated with specific antibodies at $4^{\circ} \mathrm{C}$ for $12 \mathrm{~h}$ after being placed in blocking buffer. Then, the membrane was incubated with secondary antibodies for $1 \mathrm{~h}$ at room temperature. The membrane was washed with PBS containing 0.05\% Tween-20 three times, and the expression levels of proteins were then determined with a chemiluminescence system.

\section{RNA Interference Analysis}

The sequences of small interfering RNA (siRNA) oligonucleotides targeting the MHC-I heavy chain transcript were as follows: siRNA
MHC-I he sense 5'-GGUCUUCGACCUCUUCCAUTT-3' and antisense: 5' -AUGGAAGAGGUCGA AGACCTT-3'. Scrambled siRNA (sense: 5'-UUCUCCGAACGUGUCACGUTT-3' and antisense: 5' -ACGUGACACGUUCGGAGAATT-3') was used as a negative control. The siRNA was combined with lipofectamine 2000 to form complexes before transfection into each well. The complexes were added directly to the bovine endometrial epithelial cell line (BEND), and transfection was performed when cells were attached to the well. A total of $1 \times 10^{5}$ cells/well were transfected with either siRNA MHC-I hc or negative control siRNA (GenePharma, China) in 24-well culture plates in a volume of $0.5 \mathrm{~mL}$ of serum-free DMEM. After the cells were cultivated for $24 \mathrm{~h}$, the culture solution was replaced with the one used before
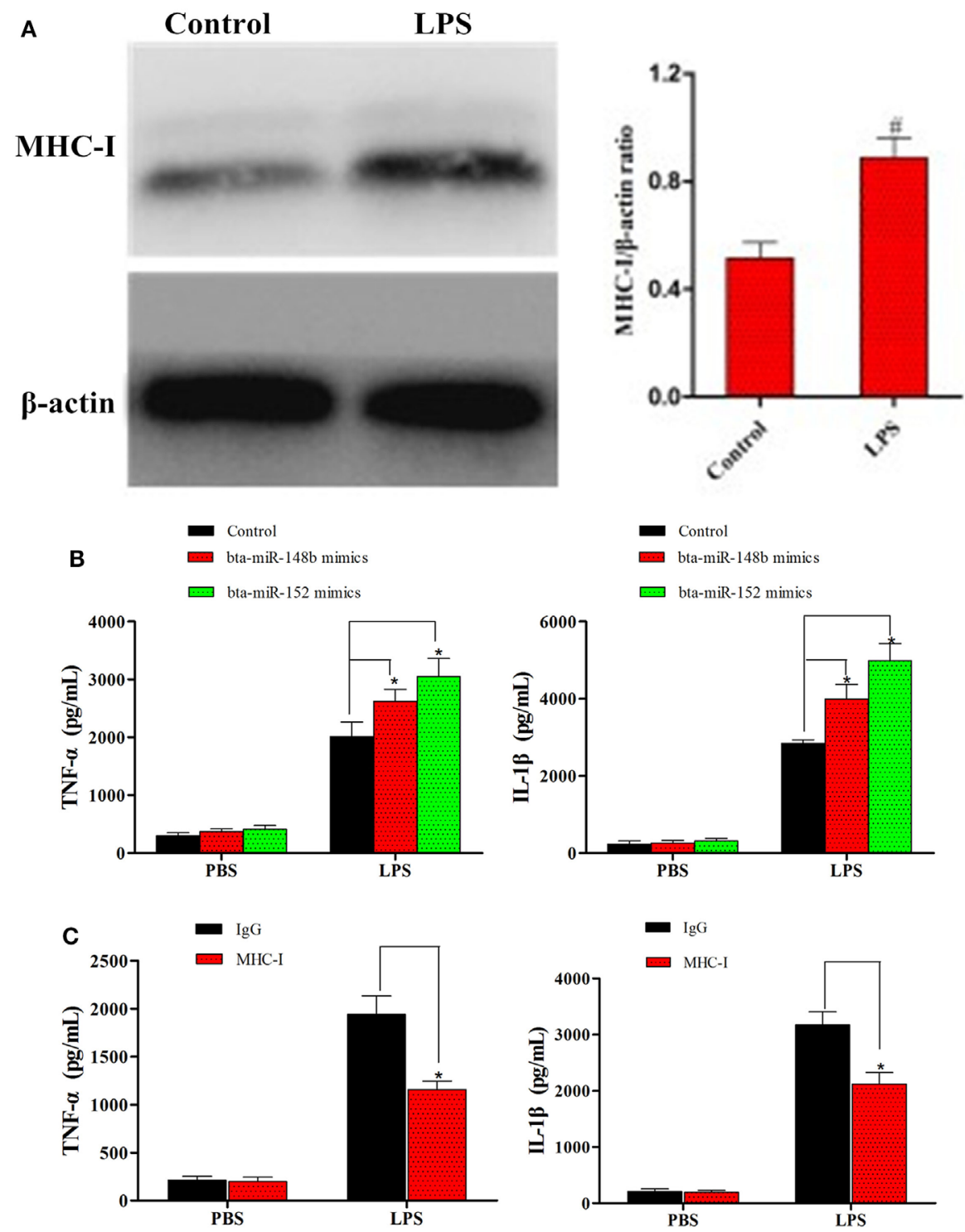

FIGURE 4 | bta-miR-148b/152 impact the regulation of MHC class I (MHC-I) molecules in TLR4-triggered inflammatory responses. (A) The expression of MHC-I was detected in lipopolysaccharide (LPS)-stimulated bovine endometrial epithelial cells (bEECs). (B) ELISA analysis of IL-1 $\beta$ and TNF- $\alpha$ in bEECs transfected with bta-miR-148b/152 mimics and then stimulated for $4 \mathrm{~h}$ with LPS. (C) ELISA analysis of IL-1 $\beta$ and TNF- $\alpha$ in bEECs after ligation with control antibody IgG or monoclonal antibodies to MHC-I and stimulation for $4 \mathrm{~h}$ with phosphate-buffered solution or LPS. Data are presented as the mean \pm SEM of three independent experiments. ${ }^{*} P<0.05$ compared to the negative control. 
transfection. After $48 \mathrm{~h}$, the cells were harvested for RNA and protein extraction.

\section{Immunofluorescence Staining Analysis}

The cells were fixed with $4 \%$ formalin for $10 \mathrm{~min}$ and then incubated with PBS containing 0.3\% Triton X-100 (Sigma, USA) and $10 \%$ bovine serum albumin to permeabilize the cells and block interactions with nonspecific proteins. Next, the cells were incubated with specific antibodies against TLR4 or p65 for $12 \mathrm{~h}$ and then with $\mathrm{Cy} 3$ secondary antibodies in the dark for $2 \mathrm{~h}$ at room temperature. Finally, TLR4 or p65 protein was detected and immobilized using mounting media containing DAPI. The cell slices were viewed under a fluorescence microscope.

\section{Statistical Analysis}

SPSS software was used for statistical analyses. Data are presented as the mean \pm SEM of three independent trials. The data were analyzed with one-way ANOVA or Student's $t$-test. $P \leq 0.05$ indicated statistically significant differences.

\section{RESULTS}

\section{Cell Identification and the Expression of the MHC-I Heavy Chain and miRNAs in IFN- $\tau$-Stimulated Cells}

Cytokeratin 18 is an epithelial-specific marker that identifies bEEC integrity. bEECs were pretreated with DAPI to identify the nucleus. The results are shown in Figure 1A. Then, we analyzed the expression of bta-miR-148b and bta-miR-152 in bEECs challenged with IFN- $\tau$ using qRT-PCR analysis. The results showed that bta-miR-148b and bta-miR-152 expression was reduced, but the level of bovine MHC-I heavy chain mRNA was increased by IFN- $\tau$ treatment (Figure 1B).

\section{bta-miR-148b and bta-miR-152 Target the Bovine MHC-I Heavy Chain Gene}

Additional assays were performed to determine whether bta-miR-148b and bta-miR-152 expression is involved in the regulation of bovine $\mathrm{MHC}-\mathrm{I}$ molecules. Using the prediction software TargetScan6.2, we found that bta-miR-148b and bta-miR-152 could potentially target the $3^{\prime}$ UTR of the MHC-I heavy chain according to the calculation of minimum free energy (Figure 2A). The miR-148b/152 sequences are predicted to interact with MHC-I 3'UTR were displayed in Figure 2B. To confirm that bta-miR-148b and bta-miR-152 directly bind the $3^{\prime}$ UTR of the bovine MHC-I heavy chain, a dual luciferase reporter assay was performed in HEK-293 cells. We integrated a fragment of the bovine MHC-I heavy chain 3'-UTR containing the target sequence or a fragment in which the target sites were mutated into the luciferase reporter vector and cotransfected this vector with bta-miR-148b mimics, bta-miR-152 mimics, or scrambled oligonucleotides. We found that bta-miR-148b/152 mimics significantly reduced the luciferase activity of wild-type MHC-I heavy chain luciferase (MHC-I heavy chain-Luc) but had no effect on mutant MHC-I heavy chain-Luc (Figure 2C). This results suggested that bta-miR-148b/152 targeted the MHC-I heavy chain gene.

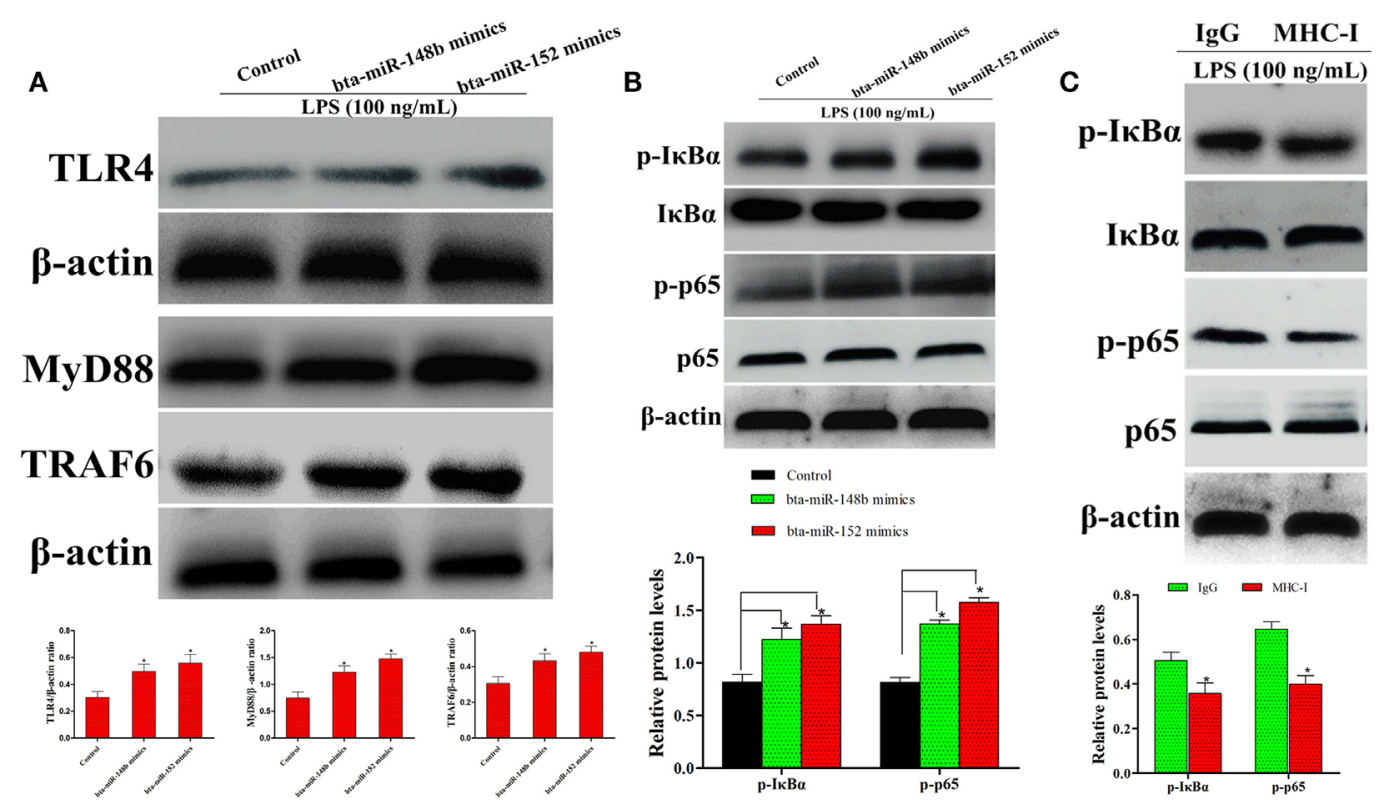

FIGURE 5 | bta-miR-148b/152 impact the regulation of MHC class I (MHC-I) molecules on TLR4 and its downstream pathway. (A) The expression of TLR4 and its downstream factors myeloid differentiation factor 88 (MyD88), TRAF6 were determined by western blot in MHC-I heavy chain-silenced bovine endometrial epithelial cells. (B) The expression of $I_{\kappa} B \alpha$ and p65 protein was determined by western blotting. Phosphorylated lkB $\alpha$ and p65 were analyzed using phospho-specific antibodies. (C) The effect of MHC-I ligation on nuclear transcription factor (NF)-kB activation was further investigated through immunoblot assay. $\beta$-actin was used as an internal control. Data are presented as the mean \pm SEM of three independent experiments. ${ }^{*} P<0.05$ compared to the negative control. 


\section{bta-miR-148b/152 Inversely Correlates with the MHC-I Heavy Chain in bEECs}

We observed that the expression of bta-miR-148b/152 increased after treatment with bta-miR-148b/152 mimics and decreased after treatment with bta-miR-148b/152 inhibitors (Figure 3A). To investigate whether bta-miR-148b/152 affect the regulation of the MHC-I heavy chain in bEECs, we measured the relative expression of the MHC-I heavy chain using immunoblotting and

A
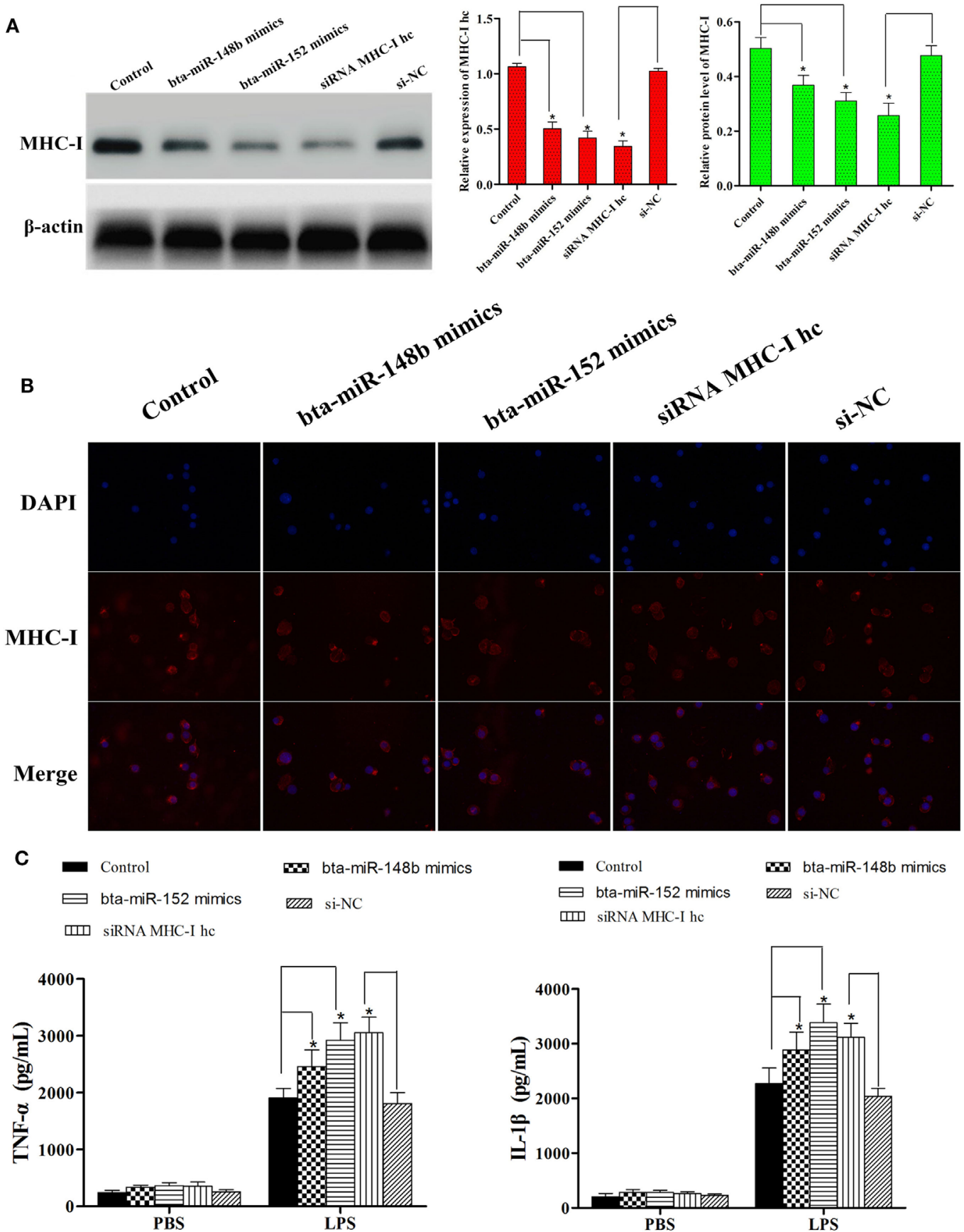

FIGURE 6 | MHC class I (MHC-I) heavy chain knockdown increases lipopolysaccharide-induced inflammatory response in BEND cells. (A) The cell line was transfected with bovine $\mathrm{MHC}-\mathrm{I}$ heavy chain small interfering RNA (siRNA), bta-miR-148b/152 mimics, or control siRNA; then, the expression of MHC-I heavy chain was detected by qRT-PCR and Western blot assays. $\beta$-actin was used as an internal control. (B) The expression of MHC-I heavy chain was further determined by immunofluorescence method. (C) The cell line was transfected with bovine MHC-I heavy chain siRNA, bta-miR-148b/152 mimics, or control siRNA; then, the expression of IL-1 $\beta$ and TNF- $\alpha$ was determined by ELISA in MHC-I heavy chain-silenced bovine endometrial epithelial cells. Data are presented as the mean \pm SEM of three independent experiments. ${ }^{*} P<0.05$ compared to the negative control. 
qRT-PCR methods. The results showed that MHC-I heavy chain expression was significantly decreased by bta-miR-148b/152 mimics but was increased by bta-miR-148b/152 inhibitors (Figures 3B,C). Thus, the data above indicated that bta-miR148b/152 negatively affected MHC-I heavy chain expression.

\section{bta-miR-148b/152 Impact the Regulation of MHC-I Molecules in TLR4-Triggered Inflammatory Responses}

Although some studies on MHC-I have shown it alleviates TLRtriggered innate inflammatory responses (23), no research has demonstrated the effects of miRNA-mediated MHC-I molecules on TLR4-induced immune responses in bEECs. Thus, to detect whether miRNA-mediated downregulation of MHC-I heavy chain expression impacts TLR4-induced inflammatory responses, LPS (a TLR4 ligand) was administered to MHC-I heavy chain-silenced bEECs. bta-miR-148b/152 mimics were used to inhibit MHC-I heavy chain expression. First, MHC-I levels were detected in cells treated or not with LPS using a western blot method. The results showed that MHC-I expression was relatively increased by LPS challenge (Figure 4A). Then, the effects of silencing the MHC-I heavy chain on cytokines was investigated by ELISA in LPSstimulated bEECs. The results showed that MHC-I heavy chain silencing increased the secretion of the pro-inflammatory mediators IL- $1 \beta$ and TNF- $\alpha$ in response to LPS challenge compared
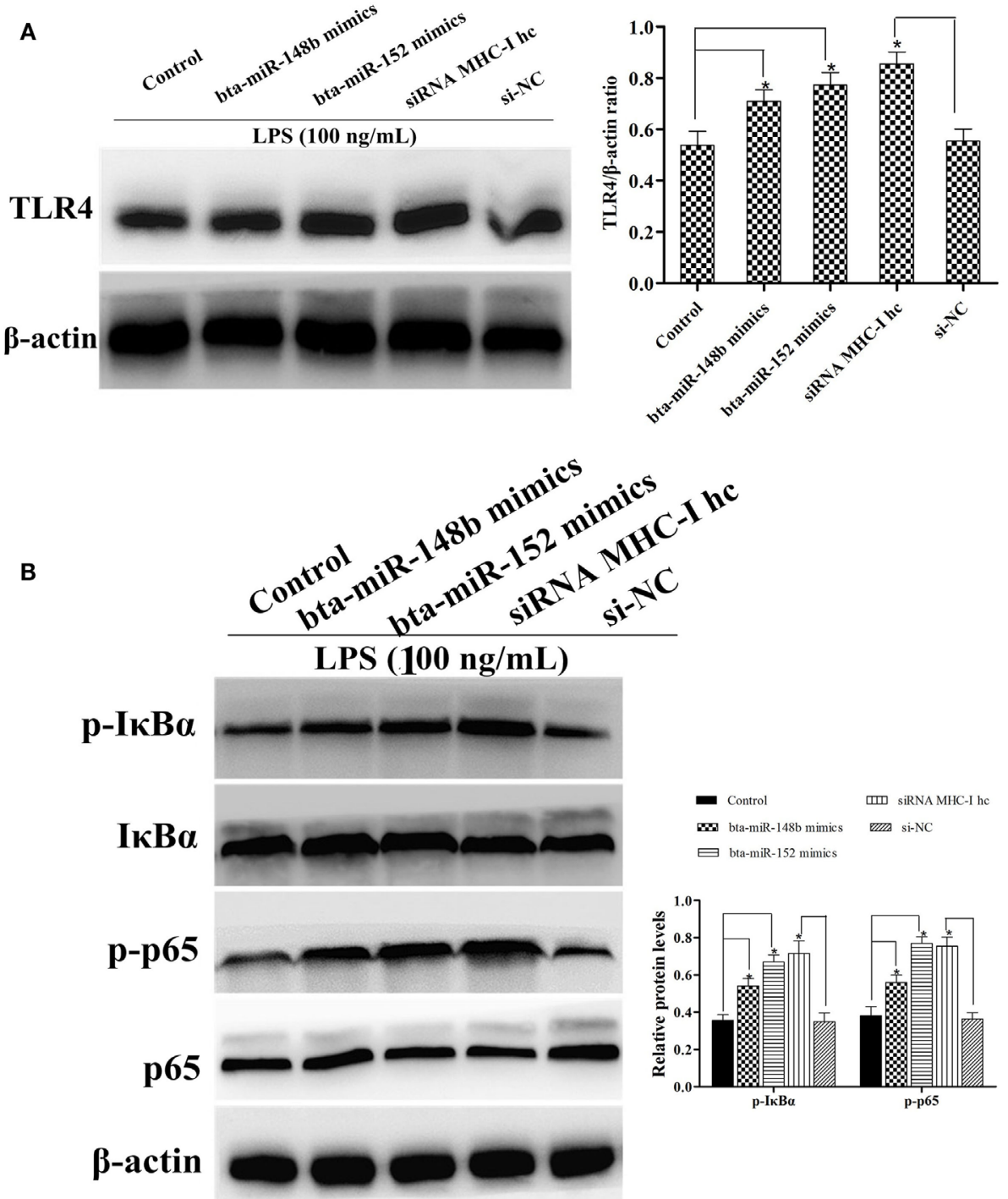

FIGURE 7 | The expression of TLR4 and its mediated signaling pathway in MHC class I (MHC-I) heavy chain knockdown BEND cells. (A) TLR4 expression level was determined by western blot assay in BEND cells. (B) The expression levels of $1 \kappa B \alpha$ and p65 protein were detected by a western blot assay in lipopolysaccharide (LPS)-stimulated BEND cells exposed to specific small interfering RNAs (siRNAs), bta-miR-148b/152 mimics, or control vectors. Phosphorylated IkB $\alpha$ and p65 were analyzed using phospho-specific antibodies. $\beta$-actin was used as an internal control. Data are presented as the mean \pm SEM of three independent experiments. ${ }^{\star} P<0.05$ compared to the negative control. 
with control mimics (Figure 4B). Moreover, cross-linking of MHC-I molecules on bEECs showed that cells crosslinked with antibodies to MHC-I (anti-HLA class I) produced lower amounts of pro-inflammatory cytokines (TNF- $\alpha$, IL-1 $\beta$ ) than did cells treated with control antibodies (Figure 4C). These data indicated that silencing of the MHC-I heavy chain by bta-miR-148b/152 enhanced LPS-induced inflammatory responses in bEECs.

\section{bta-miR-148b/152 Impacts the Regulation of MHC-I Molecules on TLR4 and Its Downstream Pathway Activation}

It is well known that LPS is a TLR4 agonist. As shown by western blot assay, compared to the control group, MHC-I heavy chainsilenced bEECs showed increased TLR4 expression after LPS stimulation. MyD88 and TRAF6 are the TLR4 signaling downstream molecules. Therefore, we also investigated the expression of these adaptor molecules in MHC-I heavy chain-silenced bEECs through western blotting. The results showed that the expression of MyD88 and TRAF6 was increased in MHC-I heavy chain-silenced bEECs (Figure 5A).

The NF- $\kappa \mathrm{B}$ pathway, which is downstream of TLR4 signaling, plays an important role in regulating the expression of inflammatory cytokines (25). To explore whether MHC-I heavy chain silencing intersects with theTLR4 signaling pathway in bEECs, the NF- $\kappa \mathrm{B}$ pathway was analyzed by western blotting. The activation of NF- $\kappa \mathrm{B}(\mathrm{I} \kappa \mathrm{B} \alpha$ and $\mathrm{p} 65)$ was enhanced in LPS-stimulated MHC-I heavy chain-silenced bEECs (Figure 5B). As MHC-I ligation alleviates TLR4-induced inflammatory responses, the effects of MHC-I ligation on the TLR4 pathway was further investigated. The results showed that cross-linking MHC-I molecules on bEECs contributed to the reduction in $\mathrm{NF}-\kappa \mathrm{B}$ pathway activation in response to LPS challenge (Figure 5C). The above data demonstrated that bta-miR-148b/152 could enhance LPS-triggered inflammatory responses by targeting the bovine MHC-I heavy chain.

\section{MHC-I Heavy Chain Knockdown Increases the LPS-Induced Inflammatory Response in BEND Cells}

We further investigated the effects of knocking down the MHC-I heavy chain on the LPS-stimulated inflammatory process in the BEND. siRNA specific to the bovine MHC-I heavy chain and btamiR-148b/152 mimics both markedly decreased the expression of the MHC-I heavy chain (Figures 6A,B). The results indicated that the MHC-I heavy chain was silenced by specific siRNA and bta-miR-148b/152 mimics.

After validating MHC-I heavy chain silencing, secretion of the pro-inflammatory cytokines IL- $1 \beta$ and TNF- $\alpha$ was determined with the ELISA method. As shown in Figure 6C, knockdown of the MHC-I heavy chain increased the production of TNF- $\alpha$ and IL-1 $\beta$, compared with control siRNA or control mimics treatment.

\section{MHC-I Heavy Chain Knockdown Enhanced the Expression of TLR4 and Activated the NF-кB Pathway in BEND Cells}

To further corroborate the result that LPS stimulation increased the expression ofTLR4 and NF- $\kappa$ B pathway activation in MHC-I heavy chain-silenced bEECs, we explored MHC-I molecule function during LPS stimulation of BEND cells using western blotting. BEND cells in which the gene encoding the MHC-I heavy chain was silenced by specific siRNA or those treated with bta-miR-148b/152 mimics showed a greater increase in TLR4 expression in response to LPS challenge than those transfected with control siRNA or control mimics (Figure 7A). The activation of the NF- $\kappa$ B pathway was determined in LPS-stimulated BEND cells with specific siRNA, bta-miR-148b/152 mimics, or the control vector through western blotting. As shown by western blot assay, the phosphorylation of $\mathrm{I} \kappa \mathrm{B} \alpha$ and $\mathrm{p} 65$ protein levels were also increased by deleting the MHC-I heavy chain in BEND cells (Figure 7B).

\section{MHC-I Heavy Chain Knockdown Promoted TLR4 Signaling in BEND Cells}

Moreover, further studies were performed to determine TLR4 and NF- $\kappa \mathrm{B}$ p 65 expression in MHC-I heavy chain gene knockout BEND cells that had been challenged with LPS via an immunofluorescence assay. We found that TLR4 (red) and p65 (green) expression both increased upon deleting MHC-I heavy chain in BEND cells (Figure 8). The results suggested that bta-miR148b/152 could promote TLR4-triggered inflammatory responses by targeting the bovine MHC-I heavy chain.

\section{MHC-I Suppressed TLR4 Pathway by Fps Signaling Activation}

It has been reported that MHC-I molecule negatively regulated TLR-induced inflammatory reactions through the Fps-SHP-2 pathway (23). Thus, the potential mechanism of the inhibition of TLR4 pathway via MHC-I molecule was further investigated. As displayed in Figure 9A, the results displayed that MHC-I molecule

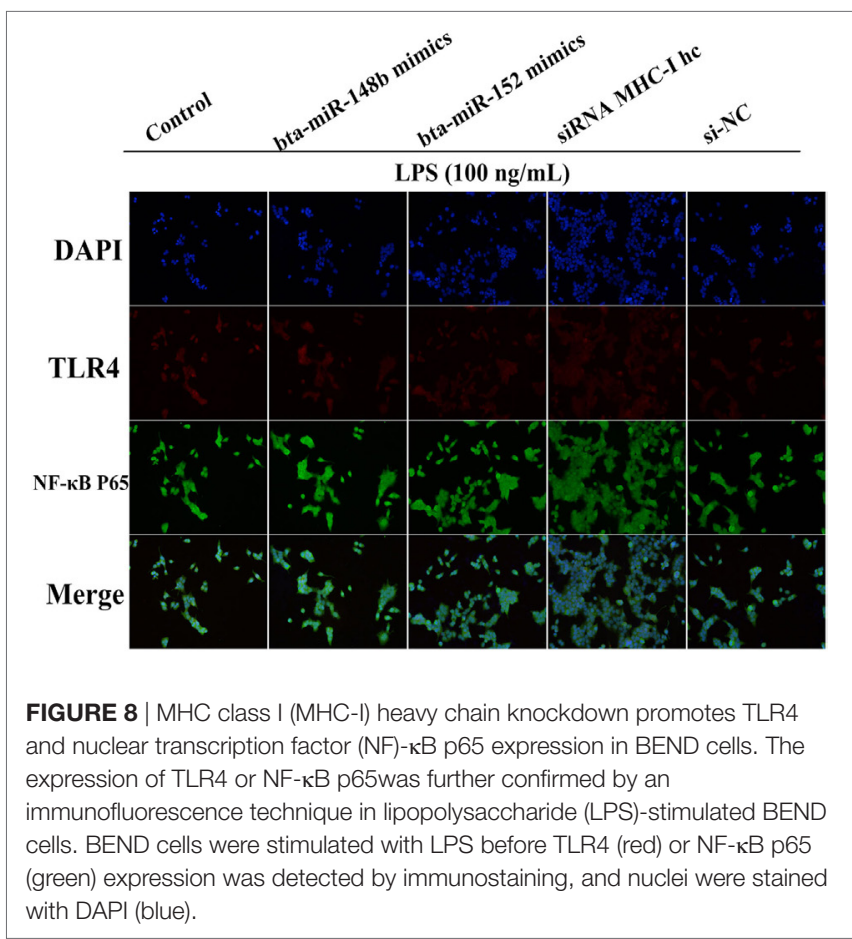



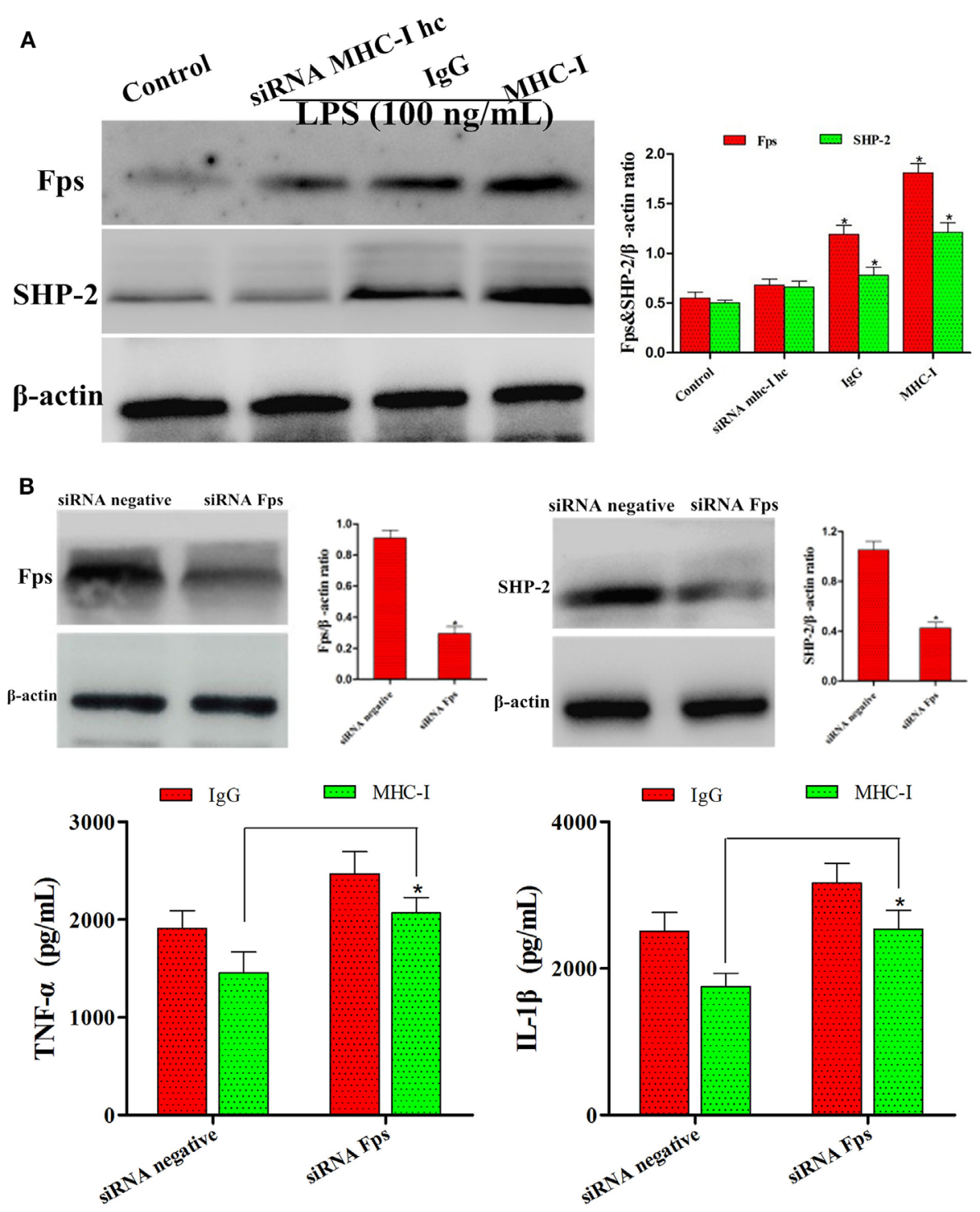

FIGURE 9 | MHC class I (MHC-I) suppressed TLR4 pathway via Fps signaling activation. (A) The expression of Fps and SHP-2 were determined by immunoblot assay. (B) Immunoblot analysis of Fps and SHP-2 after transfected with control small interfering RNA (siRNA) or Fps siRNA, and then detected the inflammatory reactions by ELISA kits. $\beta$-actin was used as an internal control. Data are presented as the mean \pm SEM of three independent experiments. ${ }^{\star} P<0.05$ compared to the negative control.

crosslinkage enhanced its interaction with Fps. Besides, after stimulation with LPS, the SHP-2 phosphorylation was reduced in which the gene encoding Fps was interfered and then enhanced the pro-inflammatory cytokines secretions (Figure 9B). These above results indicated that, and the MHC-I molecule negatively regulated TLR4-induced inflammatory reactions may through enhancing the Fps-SHP-2 pathway activation.

\section{DISCUSSION}

IFN- $\tau$ is well known as a vital pregnancy cytokine and is secreted by trophoblast cells during implantation (26). It has been reported that many of the immune response genes are under the control of IFN- $\tau$ in bEECs (27). Evidence accumulated so far strongly suggests that IFN- $\tau$ plays a vital role in the early immunological interactions between the maternal-fetal interface, exerting regulatory effects of MHC molecules on cellular expression $(2,10)$. In this experiment, we observed that IFN- $\tau$ promoted MHC-I expression and then alleviated the inflammatory response in bEECs. miRNA, or non-coding small RNA, have become known as novel molecular regulators of numerous genes and pathways involved in immune responses (28). Several studies have demonstrated that miR-148/152 is associated with the immune process $(15,29,30)$. Thus, we believe that IFN- $\tau$ regulates MHC-I molecule expression and function, possibly by affecting expression of some specific miRNAs. In the present study, we observed that bta-miR-148b/152 were inversely correlated with the MHC-I heavy chain in IFN- $\tau$-stimulated bEECs. 
MHC class I molecules play a vital role in the immune system, and the high diversity of these genes makes it possible for populations to survive in the presence of rapidly developing pathogens (31). It has been found that MHC-I class molecule (BoLA-I) antigens are associated with the occurrence of clinical mastitis $(32,33)$. Moreover, TLR is the main regulator of the initiation of the innate immune response, which perceives microorganism infection and triggers various antibacterial and inflammatory reactions (34). A previous study showed that MHC-I molecules are engaged in cross talk with TLR-triggered inflammatory responses (23). In addition, Liu et al. also showed that miR148/152 can inhibit TLR-triggered MHC-II expression, which may act as a fine-tuner in the regulation of immune responses (14). Accordingly, we hypothesized that bta-miR-148b/152 might be involved in the regulation of MHC-I and TLR receptors.

TLR4, as a major TLR receptor, specifically identifies LPS, and its activation initiates intracellular signaling, including the

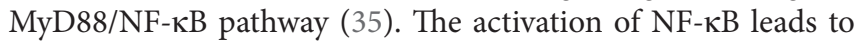
secretion of the pro-inflammatory mediators TNF- $\alpha$ and IL- $1 \beta$ (36). IL-1 $\beta$ and TNF- $\alpha$ are usually considered pro-inflammatory mediators and have important roles in the immunologic system (37). In the current research, we found that knockdown of the MHC-I heavy chain increased the expression of the proinflammatory mediators TNF- $\alpha$ and IL- $1 \beta$ in LPS-stimulated bEECs. These results indicated that MHC-I deficiency increased TLR4-induced inflammatory reactions in bEECs.

Nuclear transcription factor- $\kappa \mathrm{B}$ controls a broad spectrum of target gene expression, as a key coordinator of inflammatory and immune responses, as well as cell differentiation, proliferation, apoptosis, and survival $(38,39)$. The present study showed that NF- $\kappa$ B activation was enhanced in LPS-stimulated, MHC-I heavy chain-silenced bEECs. Moreover, Fps, a non-receptor tyrosine kinase, has vital roles in the process of inflammation and immunity (40). SHP-2 as a downstream factor of Fps and its expression pattern is similar to that of MHC-I molecules $(23,41)$. The present results suggested that Fps associated with SHP-2 and that this association inhibited the TLR4 responses by MHC-I molecule. Our data provide evidence that the MHC-I molecule negatively regulated TLR4-induced inflammatory reactions may through the Fps-SHP-2 pathway.

In conclusion, as illustrated in Figure 10, our data demonstrated that IFN- $\tau$ promoted MHC-I expression and then alleviated the inflammatory response in bEECs, and the mechanism is through affecting the expression of bta-miR-148b/152. Furthermore, we also found that bta-miR-148b/152 could

\section{REFERENCES}

1. Wu H, Zhao G, Jiang K, Chen X, Rui G, Qiu C, et al. IFN-tau alleviates lipopolysaccharide-induced inflammation by suppressing NF-kappaB and MAPKs pathway activation in mice. Inflammation (2016) 39(3):1141-50. doi:10.1007/s10753-016-0348-9

2. Chelmońskasoyta A. Interferon tau and its immunobiological role in ruminant reproduction. Arch Immunol Ther Exp (Warsz) (2002) 50(1): 47-52.

3. Chon TW, Bixler S. Interferon-tau: current applications and potential in antiviral therapy. J Interferon Cytokine Res (2010) 30(7):477-85. doi:10.1089/ jir.2009.0089

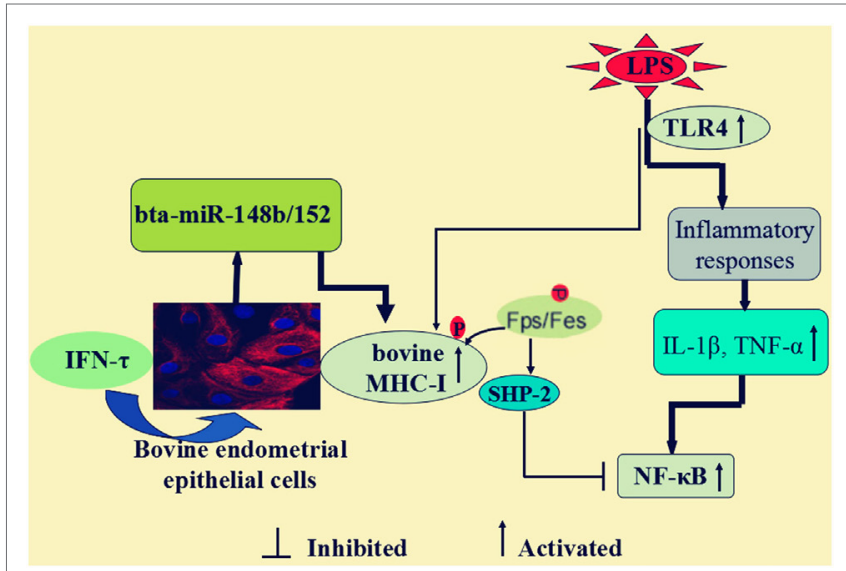

FIGURE 10 | Scheme summarizing the IFN- $\tau$ promoted the MHC class I (MHC-I) expression, and then alleviated the inflammatory response in lipopolysaccharide (LPS)-induced bovine endometrial epithelial cells. The mechanism was that bta-miR-148b/152 could promote TLR4-triggered inflammatory responses by targeting bovine $\mathrm{MHC}-\mathrm{I}$ heavy chain, and the $\mathrm{MHC}-\mathrm{I}$ molecule negatively regulated TLR4-induced inflammatory reactions may through the Fps-SHP-2 pathway.

promote TLR4-triggered inflammatory responses by targeting the bovine MHC-I heavy chain, and the MHC-I molecule negatively regulated TLR4-induced inflammatory reactions may through the Fps-SHP-2 pathway. Our discovery offers novel insight into the negative regulation of the TLR4 signaling pathway and elucidates the mechanism by which bovine MHC-I molecules control congenital inflammatory reactions.

\section{AUTHOR CONTRIBUTIONS}

HW and GD contributed to the conception and design of the study. HW, KJ, and SG performed experiments, collected samples, and accomplished the laboratory investigations. JY and GZ acquired data. HW, KJ, and GD conducted the data analysis. HW and KJ drafted the manuscript. HW, CQ, and GD modified the manuscript. All the authors have read and agreed to the final manuscript.

\section{FUNDING}

The present research was supported by the National Natural Science Foundation of China (Nos. 31472254 and 31772816).

4. Hara K, Shirasuna K, Usui F, Karasawa T, Mizushina Y, Kimura H, et al Interferon-tau attenuates uptake of nanoparticles and secretion of interleukin-1beta in macrophages. PLoS One (2014) 9(12):e113974. doi:10.1371/ journal.pone.0113974

5. Jiang K, Chen X, Zhao G, Wu H, Mi J, Qiu C, et al. IFN-tau plays an anti-inflammatory role in Staphylococcus aureus-induced endometritis in mice through the suppression of NF-kappaB pathway and MMP9 expression. J Interferon Cytokine Res (2017) 37(2):81-9. doi:10.1089/jir.2016.0058

6. Aarestrup FM, Jensen NE, Ostergard H. Analysis of associations between major histocompatibility complex (BoLA) class I haplotypes and subclinical mastitis of dairy cows. J Dairy Sci (1995) 78(8):1684-92. doi:10.3168/jds. S0022-0302(95)76793-5 
7. Araibi EH, Marchetti B, Dornan ES, Ashrafi GH, Dobromylskyj M, Ellis SA, et al. The E5 oncoprotein of BPV-4 does not interfere with the biosynthetic pathway of non-classical MHC class I. Virology (2006) 353(1):174-83. doi:10.1016/j.virol.2006.05.031

8. Marchetti B, Ashrafi GH, Dornan ES, Araibi EH, Ellis SA, Campo MS. The E5 protein of BPV-4 interacts with the heavy chain of MHC class I and irreversibly retains the MHC complex in the Golgi apparatus. Oncogene (2006) 25(15):2254-63. doi:10.1038/sj.onc. 1209245

9. Marchetti B, Gault EA, Cortese MS, Yuan Z, Ellis SA, Nasir L, et al. Bovine papillomavirus type 1 oncoprotein E5 inhibits equine MHC class I and interacts with equine MHC I heavy chain. J Gen Virol (2009) 90(12):2865-70. doi:10.1099/vir.0.014746-0

10. Todd I, McElveen JE, Lamming GE. Ovine trophoblast interferon enhances MHC class I expression by sheep endometrial cells. J Reprod Immunol (1998) 37(2):117-23. doi:10.1016/S0165-0378(97)00074-0

11. Wu H, Zhang T, Ma X, Jiang K, Zhao G, Qiu C, et al. Specific microRNA library of IFN-tau on bovine endometrial epithelial cells. Oncotarget (2017) 8(37):61487-98. doi:10.18632/oncotarget.18470

12. Bartel DP. MicroRNAs: genomics, biogenesis, mechanism, and function. Cell (2004) 116(2):281-97. doi:10.1016/S0092-8674(04)00045-5

13. Chen T, Huang Z, Wang L, Wang Y, Wu F, Meng S, et al. MicroRNA-125a-5p partly regulates the inflammatory response, lipid uptake, and ORP9 expression in oxLDL-stimulated monocyte/macrophages. Cardiovasc Res (2009) 83(1):131. doi:10.1093/cvr/cvp121

14. Liu X, Zhan Z, Xu L, Ma F, Li D, Guo Z, et al. MicroRNA-148/152 impair innate response and antigen presentation of TLR-triggered dendritic cells by targeting CaMKII $\alpha$. J Immunol (2010) 185(12):7244-51. doi:10.4049/ jimmunol.1001573

15. Manaster I, Goldmanwohl D, Greenfield C, Nachmani D, Tsukerman P, Hamani Y, et al. miRNA-mediated control of HLA-G expression and function. PLoS One (2012) 7(3):e33395. doi:10.1371/journal.pone.0033395

16. Romagne F. Current and future drugs targeting one class of innate immunity receptors: the toll-like receptors. Drug Discov Today (2007) 12(1-2):80-7. doi:10.1016/j.drudis.2006.11.007

17. Kawai $\mathrm{T}$, Akira $\mathrm{S}$. The role of pattern-recognition receptors in innate immunity: update on toll-like receptors. Nat Immunol (2010) 11(5):373. doi:10.1038/ni.1863

18. Takeda K, Kaisho T, Akira S. Toll-like receptors. Annu Rev Immunol (2003) 21(1):335-76. doi:10.1146/annurev.immunol.21.120601.141126

19. Noreen M, Arshad M. Association of TLR1, TLR2, TLR4, TLR6, and TIRAP polymorphisms with disease susceptibility. Immunol Res (2015) 62(2):234-52. doi:10.1007/s12026-015-8640-6

20. Rosadini CV, Kagan JC. Early innate immune responses to bacterial LPS. Curr Opin Immunol (2017) 44:14-9. doi:10.1016/j.coi.2016.10.005

21. Molteni M, Gemma S, Rossetti C. The role of toll-like receptor 4 in infectious and noninfectious inflammation. Mediators Inflamm (2016) 2016:6978936. doi:10.1155/2016/6978936

22. Yang Y, Lv J, Jiang S, Ma Z, Wang D, Hu W, et al. The emerging role of tolllike receptor 4 in myocardial inflammation. Cell Death Dis (2016) 7:e2234. doi:10.1038/cddis.2016.140

23. Xu S, Liu X, Bao Y, Zhu X, Han C, Zhang P, et al. Constitutive MHC class I molecules negatively regulate TLR-triggered inflammatory responses via the Fps-SHP-2 pathway. Nat Immunol (2012) 13(6):551-9. doi:10.1038/ni.2283

24. Skarzynski DJ, Miyamoto Y, Okuda K. Production of prostaglandin f(2alpha) by cultured bovine endometrial cells in response to tumor necrosis factor alpha: cell type specificity and intracellular mechanisms. Biol Reprod (2000) 62(5):1116-20. doi:10.1095/biolreprod62.5.1116

25. Yang YI, Woo JH, Seo YJ, Lee KT, Lim Y, Choi JH. Protective effect of brown alga phlorotannins against hyper-inflammatory responses in lipopolysaccharide-induced sepsis models. J Agric Food Chem (2016) 64(3):570-8. doi:10.1021/acs.jafc.5b04482

26. Takahashi M, Takahashi H, Hamano S, Watanabe S, Inumaru S, Geshi M, et al. Possible role of interferon- $\tau$ on in vitro development of bovine embryos. J Reprod Dev (2003) 49(4):297-305. doi:10.1262/jrd.49.297
27. Bai H, Sakurai T, Fujiwara H. Functions of interferon tau as an immunological regulator for establishment of pregnancy. Reprod Med Biol (2012) 11(3):109-16. doi:10.1007/s12522-011-0117-2

28. Singh RP, Massachi I, Manickavel S, Singh S, Rao NP, Hasan S, et al. The role of miRNA in inflammation and autoimmunity. Autoimmun Rev (2013) 12(12):1160-5. doi:10.1016/j.autrev.2013.07.003

29. Jasinski-Bergner S, Stoehr C, Bukur J, Massa C, Braun J, Huttelmaier S, et al. Clinical relevance of miR-mediated HLA-G regulation and the associated immune cell infiltration in renal cell carcinoma. Oncoimmunology (2015) 4(6):e1008805. doi:10.1080/2162402x.2015.1008805

30. Bian X, Si Y, Zhang M, Wei R, Yang X, Ren H, et al. Down-expression of miR-152 lead to impaired anti-tumor effect of NK via upregulation of HLA-G. Tumour Biol (2016) 37(3):3749-56. doi:10.1007/s13277-015-3669-7

31. Ellis SA, Hammond JA. The functional significance of cattle major histocompatibility complex class I genetic diversity. Annu Rev Anim Biosci (2014) 2(2):285. doi:10.1146/annurev-animal-022513-114234

32. Oddgeirsson O, Simpson SP, Morgan AL, Ross DS, Spooner RL. Relationship between the bovine major histocompatibility complex (BoLA), erythrocyte markers and susceptibility to mastitis in Icelandic cattle. Anim Genet (1988) 19(1):11-6. doi:10.1111/j.1365-2052.1988.tb00783.x

33. Sharif S, Mallard BA, Wilkie BN, Sargeant JM, Scott HM, Dekkers JC, et al. Associations of the bovine major histocompatibility complex DRB3 (BoLADRB3) alleles with occurrence of disease and milk somatic cell score in Canadian dairy cattle. Anim Genet (1998) 29(3):185-93. doi:10.1111/j.13652052.1998.00318.x

34. Pascual M, Fernandez-Lizarbe S, Guerri C. Role of TLR4 in ethanol effects on innate and adaptive immune responses in peritoneal macrophages. Immunol Cell Biol (2011) 89(6):716-27. doi:10.1038/icb.2010.163

35. Vaure C, Liu Y. A comparative review of toll-like receptor 4 expression and functionality in different animal species. Front Immunol (2014) 5:316. doi:10.3389/fimmu.2014.00316

36. Li W, Fu K, Lv X, Wang Y, Wang J, Li H, et al. Lactoferrin suppresses lipopolysaccharide-induced endometritis in mice via down-regulation of the NF-kappaB pathway. Int Immunopharmacol (2015) 28(1):695-9. doi:10.1016/j. intimp.2015.07.040

37. Salles v.J, Mázaladeoliveira T, Cândida d.R.O.A, Granja MG, Gonçalvesdealbuquerque CF, Castrofarianeto HC, et al. The trophic effect of ouabain on retinal ganglion cells is mediated by IL- $1 \beta$ and TNF- $\alpha$. Biochem Biophys Res Commun (2016) 478(1):378-84. doi:10.1016/j.bbrc.2016.07.043

38. Toscano MA, Campagna L, Molinero LL, Cerliani JP, Croci DO, Ilarregui JM, et al. Nuclear factor (NF)-kappaB controls expression of the immunoregulatory glycan-binding protein galectin-1. Mol Immunol (2011) 48(15-16): 1940-9. doi:10.1016/j.molimm.2011.05.021

39. Ma B, Hottiger MO. Crosstalk between Wnt/ $\beta$-catenin and NF- $\mathrm{KB}$ signaling pathway during inflammation. Front Immunol (2016) 7(3):378. doi:10.3389/ fimmu.2016.00378

40. Greer P. Closing in on the biological functions of Fps/Fes and Fer. Nat Rev Mol Cell Biol (2002) 3(4):278-89. doi:10.1038/nrm783

41. Lorenz U. SHP-1 and SHP-2 in T cells: two phosphatases functioning at many levels. Immunol Rev (2009) 228(1):342-59. doi:10.1111/j.1600-065X.2008. 00760.x

Conflict of Interest Statement: The authors declare that the research was conducted in the absence of any commercial or financial relationships that could be construed as a potential conflict of interest.

Copyright $\odot 2018$ Wu, Jiang, Guo, Yang, Zhao, Qiu and Deng. This is an open-access article distributed under the terms of the Creative Commons Attribution License (CC BY). The use, distribution or reproduction in other forums is permitted, provided the original author(s) and the copyright owner are credited and that the original publication in this journal is cited, in accordance with accepted academic practice. No use, distribution or reproduction is permitted which does not comply with these terms. 